\title{
Controllability results for a class of one-dimensional degenerate parabolic problems in nondivergence form
}

\author{
PieRMARCo CANNARSA, GENNI Fragnelli, and DARIo RoCCHETTI
}

Abstract. We give null controllability results for some degenerate parabolic equations in non divergence form on a bounded interval. In particular, the coefficient of the second order term degenerates at the extreme points of the domain. For this reason, we obtain an observability inequality for the adjoint problem. Then we prove Carleman estimates for such a problem. Finally, in a standard way, we deduce null controllability also for semilinear equations.

\section{Introduction}

During the last forty years controllability problems for parabolic equations has been widely investigated (see, e.g., [2],[15] and [28]). In particular, the case of uniformly parabolic equations is well-understood: for all $T>0$ there exists a control $f \in L^{2}((0, T) \times$ $(0,1))$ such that the solution of

$$
\begin{cases}u_{t}-a(x) u_{x x}=f(t, x) \chi_{\omega}(x), & (t, x) \in(0, T) \times(0,1), \\ u(t, 0)=u(t, 1)=0, & t \in(0, T), \\ u(0, x)=u_{0}(x), & x \in(0,1),\end{cases}
$$

satisfies $u(T, x)=0$ for all $x \in[0,1]$. Here, $a$ is a positive continuous function on $[0,1]$ and $\chi_{\omega}$ is the characteristic function of a given non-empty interval $\omega=(\alpha, \beta) \subset \subset[0,1]$. The reader is referred to [14] for a seminal paper in this research direction, and to [20] and [30] for the approach based on Carleman estimates. Several results have also been obtained for semilinear nondegenerate equations, see, in particular, [14], [19], [20], [22].

However, many problems that are relevant for applications are described by degenerate equations, with degeneracy occurring at the boundary of the space domain. For instance, degenerate parabolic equations can be obtained by suitable transformations from the Prandtl equations, see [25]. In a different context, degenerate operators have been extensively studied since Feller's investigations in [17], [18], where the main motivation was the rilevance of the previous problem in transition probabilities.

Mathematics Subject Classification (2000). 93B05, 93C20, 93B07, 35K65.

Key words: Degenerate parabolic equations, null controllability, Carleman estimates, Hardy type inequality. 
Recently, null controllability for degenerate parabolic equations in divergence form has been the subject of several papers. In [5] and [6] the regional null controllability of

$$
\begin{cases}u_{t}-\left(a(x) u_{x}\right)_{x}=f(t, x) \chi_{\omega}(x), & (t, x) \in(0, T) \times(0,1), \\ u(t, 0)=u(t, 1)=0, & t \in(0, T), \\ u(0, x)=u_{0}(x), & x \in(0,1),\end{cases}
$$

has been studied directly, using a technique based on cut off functions. In [1], [7], [8], [9] and [24] observability results for the adjoint problem of (1.2) are obtained using Carleman estimates. Then, the null controllability of $(1.2)$ on the full interval $[0,1]$ is derived by standard arguments. Several results have also been obtained for a semilinear version of (1.2), see, for example, [1], [4] or [5].

One of the main goals of this paper is to provide a full analysis of the null controllability problem for (1.1) when $a$ degenerates at $x=0$ and $x=1$, satisfying suitable assumptions near these points (see Hypothesis 3.1). For example, an interesting model problem of (1.1) is the one with

$$
a(x)=x^{K_{1}}(1-x)^{K_{2}}, \quad K_{1}, K_{2} \in(0,2) .
$$

Indeed, for $K_{1}=K_{2}=1$ and $f=0,(1.1)$ is related to the Feller semigroup associated to the Wright-Fischer diffusion process in [0, 1] (see, e.g., [21], [23] and [29]). We observe that the restriction $K_{1}, K_{2} \in(0,2)$ is natural if we want to obtain global null controllability: if $K_{1}$ or $K_{2} \geq 2$, then the model fails to be null controllable (see Remark 4.6).

Let us now explain the main difference as far as controllability is concerned, between degenerate parabolic equations in divergence form such as (1.2) and our problem (1.1). Clearly, equation (1.1) can be recast in divergence form as follows

$$
u_{t}-\left(a(x) u_{x}\right)_{x}+a_{x}(x) u_{x}=f(t, x) \chi_{\omega}(x)
$$

at the price of adding the drift term $a_{x} u_{x}$. Such an addition has major consequences. Indeed, as described in [3], degenerate equations of the form (1.4), are well-posed in $L^{2}(0,1)$ under the structural assumption

$$
\left|a_{x}(x)\right| \leq C \sqrt{a(x)}
$$

where $C$ is a positive constant. Observe that we need well-posedness in $L^{2}(0,1)$ since the results of [24] for (1.2) are obtained in such a space. Now, imposing (1.5) on $a_{x}$ for $a(x)=x^{K}$ gives $K \geq 2$. So, in view of the above considerations, the conditions that ensure that (1.4) is well-posed prevent (1.4) from being null-controllable. Another difference between (1.2) and (1.1) is the fact that the natural space to study (1.2) is $L^{2}(0,1)$ whereas (1.1) is more conveniently set in the weighted space 


$$
L_{\frac{1}{a}}^{2}(0,1):=\left\{u \in L^{2}(0,1) \mid \int_{0}^{1} u^{2} \frac{1}{a} d x<\infty\right\} .
$$

In such a space, (1.1) is well-posed and operator $a u_{x x}$ turns out to be self-adjoint, see [3], [16] and section 2 of this article for details.

The main technical part of the paper is, as in [24], the analysis of the adjoint problem of (1.1). In order to decouple the difficulties related to degeneracy at 0 and 1 , fixed $h \in$ $L^{2}\left((0, T) ; L_{\frac{1}{a}}^{2}(0,1)\right)$, we consider two parabolic problems in two different domains, namely

$$
\left(P_{i}\right)_{i=1,2} \begin{cases}v_{t}+a(x) v_{x x}=h(t, x), & (t, x) \in Q_{i}:=(0, T) \times J_{i}, \\ v\left(t, \partial J_{i}\right)=0, & t \in(0, T),\end{cases}
$$

where $J_{1}:=\left(0, j_{1}\right)$ and $J_{2}:=\left(j_{2}, 1\right)$ are proper subintervals of $(0,1)$. Notice that, for both $\left(P_{1}\right)$ and $\left(P_{2}\right) a$ degenerates only at one point. We then derive, for any (sufficiently smooth) solution $v$ of $\left(P_{i}\right)_{i=1,2}$, the Carleman estimates

$$
\begin{aligned}
\int_{Q_{1}}\left(s \theta v_{x}^{2}+s^{3} \theta^{3}\left(\frac{x}{a}\right)^{2} v^{2}\right) e^{2 s \varphi_{1}} d x d t \leq & C \int_{Q_{1}} h^{2} \frac{e^{2 s \varphi_{1}}}{a} d x d t \\
& +2 s C \int_{0}^{T} \theta(t)\left[x v_{x}^{2} e^{2 s \varphi_{1}}\right]\left(t, j_{1}\right) d t
\end{aligned}
$$

and

$$
\begin{aligned}
\int_{Q_{2}}\left(s \theta v_{x}^{2}+s^{3} \theta^{3}\left(\frac{x-1}{a}\right)^{2} v^{2}\right) e^{2 s \varphi_{2}} d x d t \leq & C \int_{Q_{2}} h^{2} \frac{e^{2 s \varphi_{2}}}{a} d x d t \\
& +2 s C \int_{0}^{T} \theta(t)\left[(1-x) v_{x}^{2} e^{2 s \varphi_{2}}\right]\left(t, j_{2}\right) d t,
\end{aligned}
$$

for all $s \geq s_{0}$ ( $s_{0}$ being a suitable constant), where $\theta(t), \varphi_{1}(t, x)$ and $\varphi_{2}(t, x)$ are suitable weight functions.

Thanks to (1.6) and (1.7), we derive the observability inequality

$$
\int_{0}^{1} v^{2}(0, x) \frac{1}{a} d x \leq C \int_{0}^{T} \int_{\omega} v^{2} \frac{1}{a} d x d t
$$

for any solution of the adjoint problem of (1.1). Then the null controllability of (1.1) follows in a standard way. 
Observe that an essential tool of this approach is Hardy's inequality

$$
\int_{0}^{1} v^{2} \frac{d x}{x^{2}} \leq C \int_{0}^{1} v_{x}^{2} d x \quad \forall v \in H_{0}^{1}(0,1),
$$

where $C$ is a positive constant (see, e.g., [10]).

Finally, we derive analogous results for equations with a bounded potential term

$$
\left\{\begin{array}{l}
u_{t}-a(x) u_{x x}+b(t, x) u=f(t, x) \chi_{\omega}(x) \\
u(t, 0)=u(t, 1)=0 \\
u(0, x)=u_{0}(x)
\end{array}\right.
$$

Such a generalization allows to obtain, arguing as in [19], [1], the null controllability for the semilinear problem

$$
\begin{cases}u_{t}-a(x) u_{x x}+h(t, x, u)=f(t, x) \chi_{\omega}(x), & (t, x) \in(0, T) \times(0,1), \\ u(t, 0)=u(t, 1)=0, & t \in(0, T), \\ u(0, x)=u_{0}(x), & x \in(0,1) .\end{cases}
$$

The paper is organized as follows:

- in Section 2., we prove the well-posedness of the linear problem (1.1);

- in Section 3, we state Carleman estimates for the adjoint problems $\left(P_{i}\right)$;

- in Section 4, we prove the observability inequality for the adjoint problem (1.1) and, as a consequence, we give a null controllability result for (1.1);

- in Section 5., we extend the previous results to the generalized linear problem (1.10) and to the semilinear problem (1.11).

\section{Well-posedness}

Let $T>0,{ }_{\wedge} Q:=(0, T) \times(0,1), \alpha:=(\alpha, \beta) \subset \subset(0,1)$ be a non-empty assigned interval and the data $\left(f, u_{0}\right) \in L^{2}(Q) \times L_{\frac{1}{a}}^{2}(0,1)$, where

$$
L_{\frac{1}{a}}^{2}(0,1):=\left\{u \in L^{2}(0,1) \mid\|u\|_{\frac{1}{a}}<\infty\right\}, \quad\|u\|_{\frac{1}{a}}^{2}:=\int_{0}^{1} u^{2} \frac{1}{a} d x_{\overline{\bar{\curlywedge}}}
$$

we consider the degenerate parabolic problem

$$
\begin{cases}u_{t}-a(x) u_{x x}=f(t, x) \chi_{\omega}(x), & (t, x) \in Q, \\ u(t, 0)=u(t, 1)=0, & t \in(0, T), \\ u(0, x)=u_{0}(x), & x \in(0,1) .\end{cases}
$$

Here $a \in C^{0}[0,1]$ is such that $a(0)=a(1)=0$ and $a>0$ on $(0,1)$. 
In order to study the well-posedness of (2.1), we introduce the following Hilbert spaces:

$$
H_{\frac{1}{a}}^{1}(0,1):=L_{\frac{1}{a}}^{2}(0,1) \cap H_{0}^{1}(0,1)
$$

and

$$
H_{\frac{1}{a}}^{2}(0,1):=\left\{u \in H_{\frac{1}{a}}^{1}(0,1) \mid a u_{x x} \in L_{\frac{1}{a}}^{2}(0,1)\right\}
$$

with the following norms

$$
\|u\|_{1, \frac{1}{a}}^{2}:=\int_{0}^{1} u^{2} \frac{1}{a} d x+\int_{0}^{1} u_{x}^{2} d x \quad \text { and } \quad\|u\|_{2, \frac{1}{a}}^{2}:=\|u\|_{1, \frac{1}{a}}^{2}+\int_{0}^{1} a u_{x x}^{2} d x .
$$

Moreover, we establish the following Green's formula:

LEMMA 2.1. For all $(u, v) \in H_{\frac{1}{a}}^{2}(0,1) \times H_{\frac{1}{a}}^{1}(0,1)$ one has

$$
\int_{0}^{1} u_{x x} v d x=-\int_{0}^{1} u_{x} v_{x} d x
$$

Proof. First, we claim that the space $H_{c}^{1}(0,1):=\left\{v \in H^{1}(0,1) \mid \operatorname{supp}\{v\} \subset(0,1)\right\}$ is dense in $H_{\frac{1}{a}}^{1}(0,1)$. Indeed, if we consider the sequence $\left(v_{n}\right)_{n \geq 4}$, where $v_{n}:=\xi_{n} v$ for a fixed function $v \in H_{\frac{1}{a}}^{1}(0,1)$ and

$$
\xi_{n}(x):= \begin{cases}0, & x \in[0,1 / n] \cup[1-1 / n, 1], \\ 1, & x \in[2 / n, 1-2 / n], \\ n x-1, & x \in(1 / n, 2 / n), \\ n(1-x)-1, & x \in(1-2 / n, 1-1 / n),\end{cases}
$$

it is easy to see that $v_{n} \rightarrow v$ in $L_{\underline{1}}^{2}(0,1)$. Moreover, one has that

$$
\begin{aligned}
\int_{0}^{1}\left(v_{n}-v\right)_{x}^{2} d x & \leq 2 \int_{0}^{1}\left(1-\xi_{n}\right)^{2} v_{x}^{2} d x+2 \int_{0}^{1}\left(\xi_{n}\right)_{x}^{2} v^{2} d x \\
& =2 \int_{0}^{1}\left(1-\xi_{n}\right)^{2} v_{x}^{2} d x+2 n^{2}\left(\int_{\frac{1}{n}}^{\frac{2}{n}} v^{2} d x+\int_{1-\frac{2}{n}}^{1-\frac{1}{n}} v^{2} d x\right) .
\end{aligned}
$$


Obviously, the first term in the last member of (2.3) converges to zero. Furthermore, since $v \in H_{0}^{1}(0,1)$, by Hölder's inequality one has that

$$
v^{2}(x) \leq x \int_{0}^{x} v_{x}^{2}(y) d y \quad \forall x \in[0,1]
$$

Therefore,

$$
n^{2} \int_{\frac{1}{n}}^{\frac{2}{n}} v^{2} d x \leq n^{2} \int_{0}^{\frac{2}{n}} v_{x}^{2} d x \int_{\frac{1}{n}}^{\frac{2}{n}} x d x \rightarrow 0, \quad \text { as } n \rightarrow \infty .
$$

Since the remaining term in (2.3) can be similarly estimated, our claim is proved.

Now, set $\Phi(v):=\int_{0}^{1}\left(u_{x} v\right)_{x} d x$, with $u \in H_{\frac{1}{a}}^{2}(0,1)$. Then, $\Phi$ is a bounded linear functional on $H_{\frac{1}{a}}^{1}(0,1)$ such that $\Phi=0$ on $H_{c}^{1}(0,1)$. Thus, $\Phi=0$ on $H_{\frac{1}{a}}^{1}(0,1)$, that is, (2.2) holds.

An immediate consequence of the previous Green's formula is the next corollary:

COROLLARY 2.2. The space $\mathcal{D}(0,1)$ is dense in $H_{\frac{1}{a}}^{1}(0,1)$.

Proof. It is sufficient to prove that if $u \in H_{\frac{1}{a}}^{1}(0,1)$ is orthogonal to $\mathcal{D}(0,1)$ then $u=0$. For all $\varphi \in \mathcal{D}(0,1)$ we have

$$
\int_{0}^{1} u \varphi \frac{d x}{a}=-\int_{0}^{1} u_{x} \varphi_{x} d x
$$

Consequently, one has that $u_{x x}=u / a$ in the distributional sense and thus $u \in H_{\frac{1}{a}}^{2}(0,1)$. By (2.2) one has that

$$
<u, v>_{1, \frac{1}{a}}=\int_{0}^{1} \frac{u}{a} v+u_{x} v_{x} d x=\int_{0}^{1} u_{x x} v+u_{x} v_{x} d x=0 \quad \forall v \in H_{\frac{1}{a}}^{1}(0,1) .
$$

Using standard techniques (see, e.g., [16]), as a consequence of (2.2) one can prove the following theorem that refines Theorems 1.1. and 1.2 by Barbu-Favini-Romanelli [3] for the case of $n=1$. 
THEOREM 2.3. The operator $(A, D(A))$ given by

$$
A u:=a u_{x x}, \quad D(A):=H_{\frac{1}{a}}^{2}(0,1)
$$

is m-dissipative and self adjoint in $L_{\frac{1}{a}}^{2}(0,1)$.

As usual, one can prove the following well-posedness theorem.

THEOREM 2.4. For all $f \in L^{2}(Q)$ and $u_{0} \in L_{\frac{1}{a}}^{2}(0,1)$, there exists a unique weak solution $u \in \mathcal{U}:=C^{0}\left([0, T] ; L_{\frac{1}{a}}^{2}(0,1)\right) \cap L^{2}\left(0, T ; H_{\frac{1}{a}}^{1}(0,1)\right)$ of (2.1). Moreover, one has

$$
\sup _{t \in[0, T]}\|u(t)\|_{L_{\frac{1}{a}}^{2}(0,1)}^{2}+\int_{0}^{T}\|u\|_{H_{\frac{1}{a}}^{1}(0,1)}^{2} d t \leq C\left(\left\|u_{0}\right\|_{L_{\frac{1}{a}}^{2}(0,1)}^{2}+\int_{0}^{T}\|f\|_{L_{\frac{1}{a}}^{2}(\omega)}^{2} d t\right),
$$

for a positive constant $C$.

Therefore, with additional assumptions on the degenerate function $a(x)$ one can prove a characterization for the space $H_{\frac{1}{a}}^{1}(0,1)$. Assume the following:

HYPOTHESES 2.5. The function $a \in C^{0}[0,1]$ is such that $a(0)=a(1)=0, a>0$ on $(0,1)$ and there exist $K_{1}, K_{2} \in(0,2)$ such that

1) the function $x \longmapsto \frac{a(x)}{x^{K_{1}}}$ is nonincreasing near zero;

2) the function $x \longmapsto \frac{a(x)}{(1-x)^{K_{2}}}$ is nondecreasing near one.

Thanks to the previous hypothesis one has that the spaces $H_{0}^{1}(0,1)$ and $H_{\frac{1}{a}}^{1}(0,1)$ coincide. To this aim, the following Hardy-Poincaré inequality is helpful.

PROPOSITION 2.6. (Hardy-Poincaré Inequality) Assume that Hypothesis 2.5 is satisfied. Then, there exists $C>0$ such that

$$
\int_{0}^{1} v^{2} \frac{1}{a} d x \leq C \int_{0}^{1} v_{x}^{2} d x \quad \forall v \in H_{0}^{1}(0,1)
$$

Proof. Observe that since $\frac{a}{x^{K}}$ is nonincreasing near 0 , then there exists a positive constant $C$ such that $a(x) \geq C x^{K_{1}}$ near 0 . Analogously, there exists $C>0$ such that $a(x) \geq C(1-x)^{K_{2}}$ near 1 . Then, for a suitable $\varepsilon>0$ and using Hardy's inequality 


$$
\begin{aligned}
\int_{0}^{1} v^{2} \frac{1}{a} d x & =\int_{0}^{\varepsilon} v^{2} \frac{1}{a} d x+\int_{\varepsilon}^{1-\varepsilon} v^{2} \frac{1}{a} d x+\int_{1-\varepsilon}^{1} v^{2} \frac{1}{a} d x \\
& \leq C \int_{0}^{1} v^{2} \frac{1}{x^{K_{1}}} d x+\int_{\varepsilon}^{1-\varepsilon} v^{2} \frac{1}{a} d x+C \int_{0}^{1} v^{2} \frac{1}{(1-x)^{K_{2}}} d x \\
& \leq C \int_{0}^{1} v^{2} \frac{1}{x^{2}} d x+\int_{\varepsilon}^{1-\varepsilon} v^{2} \frac{1}{a} d x+C \int_{0}^{1} v^{2} \frac{1}{(1-x)^{2}} d x \\
& \leq C \int_{0}^{1} v_{x}^{2} d x+\int_{\varepsilon}^{1-\varepsilon} v^{2} \frac{1}{a} d x \leq C\left(\int_{0}^{1} v_{x}^{2} d x+\int_{\varepsilon}^{1-\varepsilon} v^{2} d x\right) \\
& \leq C\left(\int_{0}^{1} v_{x}^{2} d x+\int_{0}^{1} v^{2} d x\right) .
\end{aligned}
$$

By Poincaré's inequality, it results

$$
\int_{0}^{1} v^{2} \frac{1}{a} d x \leq C \int_{0}^{1} v_{x}^{2} d x .
$$

As an immediate consequence of Propositions 2.6, one has that $\|u\|_{1, \frac{1}{a}}$ is equivalent to $\left(\int_{0}^{1} u_{x}^{2} d x\right)^{\frac{1}{2}}$. In particular, it results:

COROLLARY 2.7. The Banach spaces $H_{\frac{1}{a}}^{1}(0,1)$ and $H_{0}^{1}(0,1)$ coincide.

REMARK 2.8. The previous result also follows from point (ii) of the Lemma in [11, p. 380]. Moreover, under Hypothesis 2.5, the density result of Corollary 2.2 is also a consequence of point (i) of the same Lemma.

Observe that if the degenerate function $a$ is also differentiable in $(0,1)$ then Hypothesis 2.5 is equivalent to the following one:

HYPOTHESES 2.9. The function $a \in C^{0}[0,1]$ is such that $a(0)=a(1)=0, a>0$ on $(0,1)$ and there exist $K_{1}, K_{2} \in(0,2)$ such that

1) $\frac{x a_{x}}{a} \leq K_{1}$ near zero; 
2) $\frac{(x-1) a_{x}}{a} \leq K_{2}$ near one.

In fact, one has that

$$
\begin{aligned}
& \left(\frac{x^{K_{1}}}{a}\right)_{x}=\frac{x^{K_{1}-1}}{a}\left(K_{1}-\frac{x a_{x}}{a}\right) \geq 0 \Longleftrightarrow \frac{x a_{x}}{a} \leq K_{1} ; \\
& \left(\frac{(1-x)^{K_{2}}}{a}\right)_{x}=-\frac{(1-x)^{K_{2}-1}}{a}\left(K_{2}-\frac{(x-1) a_{x}}{a}\right) \leq 0 \Longleftrightarrow \\
& \frac{(x-1) a_{x}}{a} \leq K_{2} .
\end{aligned}
$$

\section{Carleman Estimates for Degenerate Parabolic Problems}

In this section we prove crucial estimates of Carleman's type, that will be useful to prove the observability inequality for the adjoint problem of (2.1).

\subsection{Statement of the main results}

Given $T>0, J_{1}:=\left(0, j_{1}\right)$ and $J_{2}:=\left(j_{2}, 1\right)$ proper subintervals of $(0,1)$ and $h \in$ $L^{2}\left(0, T ; L_{\frac{1}{a}}^{2}(0,1)\right)$, we consider, for $i=1,2$, the parabolic problems

$$
\begin{cases}v_{t}+a(x) v_{x x}=h(t, x), & (t, x) \in Q_{i}:=(0, T) \times J_{i} \\ v\left(t, \partial J_{i}\right)=0, & t \in(0, T)\end{cases}
$$

Here $a:[0,1] \rightarrow \mathbb{R}$ satisfies the following assumption.

HYPOTHESES 3.1. The function $a \in C^{0}[0,1] \bigcap C^{3}(0,1)$ is such that $a(0)=a(1)=$ $0, a>0$ on $(0,1)$ and there exists $\varepsilon \in(0,1)$ such that

1) the function $\frac{x a_{x}}{a} \in L^{\infty}(0, \varepsilon)$ and there exist $K_{1} \in(0,2)$ and $C_{1}>0$ such that $\frac{x a_{x}(x)}{a(x)} \leq K_{1}$ and $\left|\left(\frac{x a_{x}(x)}{a(x)}\right)_{x x}\right| \leq C_{1} \frac{1}{a(x)} \quad \forall x \in(0, \varepsilon) ;$

2) the function $\frac{(x-1) a_{x}}{a} \in L^{\infty}(1-\varepsilon, 1)$ and there exist $K_{2} \in(0,2)$ and $C_{2}>0$ such that $\frac{(x-1) a_{x}(x)}{a(x)} \leq K_{2}$ and $\left|\left(\frac{(x-1) a_{x}(x)}{a(x)}\right)_{x x}\right| \leq C_{2} \frac{1}{a(x)} \quad \forall x \in(1-\varepsilon, 1)$. 
Now, let us introduce the weight functions

$$
\left\{\begin{array}{l}
\varphi_{i}(t, x):=\theta(t)\left(p_{i}(x)-2\left\|p_{i}\right\|_{L^{\infty}\left(J_{i}\right)}\right), \quad i=1,2, \\
p_{1}(x):=\int_{0}^{x} \frac{y}{a(y)} e^{R y^{2}} d y, \quad p_{2}(x):=\int_{j_{2}}^{x} \frac{y-1}{a(y)} e^{R(y-1)^{2}} d y, \quad R>0, \\
\theta(t):=\frac{1}{[t(T-t)]^{4}} .
\end{array}\right.
$$

Observe that $\varphi_{i}(t, x)<0 \quad \forall(t, x) \in Q_{i}, \varphi_{i}(t, x) \rightarrow+\infty$ as $t \rightarrow 0^{+}, T^{-}$and, by the assumptions on $a$, one has that $p_{i} \in C^{4}(0,1)$ (for $i=1,2$ ). Therefore, the functions $p_{i}$ are more regular as we can see from the next lemma.

LEMMA 3.2. Assume that Hypothesis 2.5 is satisfied. Then $p_{1} \in W^{1,1}\left(J_{1}\right)$ and $p_{2} \in$ $W^{1,1}\left(J_{2}\right)$.

Proof. By hypothesis, there exist two constants $C>0$ and $\epsilon \in J_{1}$ such that $\frac{x}{a(x)} e^{R x^{2}} \leq$ $C x^{1-K} e^{R}$ for all $x \in(0, \epsilon)$. Thus $\left(p_{1}\right)_{x} \in L^{1}\left(J_{1}\right)$. Similarly one proves that $\left(p_{2}\right)_{x} \in$ $L^{1}\left(J_{2}\right)$.

Our main results are the following.

THEOREM 3.3. Assume that Hypothesis 3.1.1 is satisfied for some $\varepsilon \in(0,1)$ such that $\varepsilon<j_{1}$. Then, there exist two positive constants $C$ and $s_{0}$ such that every solution $v$ of (3.1) in

$$
\mathcal{V}_{1}:=L^{2}\left(0, T ; H_{\frac{1}{a}}^{2}\left(J_{1}\right)\right) \cap H^{1}\left(0, T ; H_{\frac{1}{a}}^{1}\left(J_{1}\right)\right)
$$

satisfies, for all $s \geq s_{0}$,

$$
\begin{aligned}
\int_{Q_{1}}\left(s \theta v_{x}^{2}+s^{3} \theta^{3}\left(\frac{x}{a}\right)^{2} v^{2}\right) e^{2 s \varphi_{1}} d x d t \leq & C \int_{Q_{1}} h^{2} \frac{e^{2 s \varphi_{1}}}{a} d x d t \\
& +2 s C \int_{0}^{T} \theta(t)\left[x v_{x}^{2} e^{2 s \varphi_{1}}\right]\left(t, j_{1}\right) d t .
\end{aligned}
$$

THEOREM 3.4. Assume that Hypothesis 3.1.2 is satisfied for some $\varepsilon \in(0,1)$ such that $1-\varepsilon>j_{2}$. Then, there exist two positive constants $C$ and $s_{0}$ such that every solution $v$ of (3.1) in

$$
\mathcal{V}_{2}:=L^{2}\left(0, T ; H_{\frac{1}{a}}^{2}\left(J_{2}\right)\right) \cap H^{1}\left(0, T ; H_{\frac{1}{a}}^{1}\left(J_{2}\right)\right)
$$


satisfies, for all $s \geq s_{0}$,

$$
\begin{aligned}
\int_{Q_{2}}\left(s \theta v_{x}^{2}+s^{3} \theta^{3}\left(\frac{x-1}{a}\right)^{2} v^{2}\right) e^{2 s \varphi_{2}} d x d t \leq & C \int_{Q_{2}} h^{2} \frac{e^{2 s \varphi_{2}}}{a} d x d t \\
& +2 s C \int_{0}^{T} \theta(t)\left[(1-x) v_{x}^{2} e^{2 s \varphi_{2}}\right]\left(t, j_{2}\right) d t .
\end{aligned}
$$

We will prove only Theorem 3.3 since the proof of Theorem 3.4 is analogous.

\subsection{Preliminary technical results}

In order to prove Theorem 3.3 the following results are necessary:

LEMMA 3.5. Assume that Hypothesis 2.5.1 is satisfied. Then

$$
\lim _{x \rightarrow 0^{+}} \frac{x^{2}}{a(x)}=0 .
$$

Proof. Since $\frac{a}{x^{K_{1}}}$ is nonincreasing near zero, there exists $\epsilon \in J_{1}$ such that

$$
a(x) \geq a(\epsilon)\left(\frac{x}{\epsilon}\right)^{K_{1}} \quad \forall x \in(0, \epsilon) .
$$

Then, the following inequality holds

$$
\frac{x^{2}}{a(x)} \leq \frac{\epsilon^{K_{1}}}{a(\epsilon)} x^{2-K_{1}} \quad \forall x \in(0, \epsilon) .
$$

Passing to the limit, the conclusion follows.

LEMMA 3.6. Assume that Hypothesis 2.5 .1 is satisfied. If $w \in H_{\frac{1}{a}}^{2}\left(J_{1}\right)$, then

$$
\lim _{x \rightarrow 0^{+}} x w_{x}^{2}(x)=0 .
$$

Proof. Let $w \in H_{\frac{1}{a}}^{2}\left(J_{1}\right)$. Then, $x w_{x}^{2} \in W^{1,1}\left(J_{1}\right)$. In fact

$$
\begin{aligned}
\int_{0}^{j_{1}}\left|\left(x w_{x}^{2}\right)_{x}\right| d x & =\int_{0}^{j_{1}}\left|w_{x}^{2}+2 x w_{x} w_{x x}\right| d x \leq \int_{0}^{j_{1}} w_{x}^{2} d x+2 \int_{0}^{j_{1}} x\left|w_{x}\right|\left|w_{x x}\right| d x \\
& \leq \int_{0}^{j_{1}} w_{x}^{2} d x+\int_{0}^{j_{1}} \frac{x^{2}}{a} w_{x}^{2} d x+\int_{0}^{j_{1}} a w_{x x}^{2} d x<+\infty .
\end{aligned}
$$

It follows that $x w_{x}^{2} \rightarrow L \geq 0$ as $x \rightarrow 0$. But, if $L>0$, then $w_{x} \notin L^{2}(0,1)$. Thus $L=0$. 
PROPOSITION 3.7. Assume that Hypothesis 3.1.1 is satisfied. Then

$$
\lim _{x \rightarrow 0^{+}} x\left(\frac{x a_{x}}{a}\right)_{x}(x)=0 .
$$

Proof. Set $\rho(x):=\frac{x a_{x}(x)}{a(x)}$, we have that

$$
\rho_{x}(x)=\rho_{x}(x+h)-\int_{x}^{x+h} \rho_{x x}(y) d y
$$

and, by assumption,

$$
x\left|\rho_{x}(x)\right| \leq x\left|\rho_{x}(x+h)\right|+C_{1}\left(x \int_{x}^{x+h} \frac{d y}{a(y)}\right)
$$

for all $x \in(0, \varepsilon)$ and $h>0$ such that $x+h \in(0, \varepsilon)$.

Since $\frac{a}{x^{K_{1}}}$ is nonincreasing then $\frac{1}{a(x)} \leq C \frac{1}{x^{K_{1}}} \leq C \frac{1}{x^{K_{1}+\delta}} \quad$ for some positive constant $\delta$ such that $K_{1}+\delta \in(0,2) \backslash\{1\}$. Thus

$$
x \int_{x}^{x+h} \frac{d y}{a(y)} \leq C x \int_{x}^{x+h} \frac{d y}{y^{K_{1}+\delta}}=C x\left[\frac{y^{1-\left(K_{1}+\delta\right)}}{1-\left(K_{1}+\delta\right)}\right]_{x}^{x+h} \rightarrow 0,
$$

as $x \rightarrow 0^{+}$and, by (3.4), we obtain the conclusion.

\subsection{Proof of Theorem 3.3}

In order to prove Theorem 3.3 we define, for $s>0$, the function

$$
w(t, x):=e^{s \varphi_{1}(t, x)} v(t, x)
$$

where $v$ is the solution of (3.1) in $\mathcal{V}_{1}$; observe that, since $v \in \mathcal{V}_{1}, w \in \mathcal{V}_{1}$. Setting, for simplicity, $\varphi:=\varphi_{1}$ and $p:=p_{1}$, one has that $w$ satisfies

$$
\begin{cases}\left(e^{-s \varphi} w\right)_{t}+a(x)\left(e^{-s \varphi} w\right)_{x x}=h(t, x), & (t, x) \in Q_{1}, \\ w(0, x)=w(T, x)=0, & x \in J_{1}, \\ w(t, 0)=w\left(t, j_{i}\right)=0, & t \in(0, T) .\end{cases}
$$

Defining $L v:=v_{t}+a v_{x x}$ and $L_{s} w:=e^{s \varphi} L\left(e^{-s \varphi} w\right)$, the equation of (3.5) can be recast as follows 


$$
L_{s} w=L_{s}^{+} w+L_{s}^{-} w=e^{s \varphi} h
$$

where

$$
\left\{\begin{array}{l}
L_{s}^{+} w:=a w_{x x}-s \varphi_{t} w+s^{2} a \varphi_{x}^{2} w \\
L_{s}^{-} w:=w_{t}-2 s a \varphi_{x} w_{x}-s a \varphi_{x x} w
\end{array}\right.
$$

Moreover, set $\left\langle u, v>_{L_{\frac{1}{a}}^{2}\left(Q_{1}\right)}:=\int_{Q_{1}} u v \frac{1}{a} d x d t\right.$, one has

$$
\left\|L_{s}^{+} w\right\|_{L_{\frac{1}{a}}^{2}\left(Q_{1}\right)}^{2}+\left\|L_{s}^{-} w\right\|_{L_{\frac{1}{a}}^{2}\left(Q_{1}\right)}^{2}+2<L_{s}^{+} w, L_{s}^{-} w>_{L_{\frac{1}{a}}^{2}\left(Q_{1}\right)}=\left\|h e^{s \varphi}\right\|_{L_{\frac{1}{a}}^{2}\left(Q_{1}\right)}^{2} .
$$

LEMMA 3.8. The following identity holds

$$
\begin{aligned}
& <L_{s}^{+} w, L_{s}^{-} w>_{L_{\frac{1}{a}}^{2}\left(Q_{1}\right)}=s \int_{Q_{1}}\left(a \varphi_{x x}+\left(a \varphi_{x}\right)_{x}\right) w_{x}^{2} d x d t \\
& +s^{3} \int_{Q_{1}} \varphi_{x}^{2}\left(a \varphi_{x x}+\left(a \varphi_{x}\right)_{x}\right) w^{2} d x d t \\
& -2 s^{2} \int_{Q_{1}} \varphi_{x} \varphi_{x t} w^{2} d x d t+\frac{s}{2} \int_{Q_{1}} \frac{\varphi_{t t}}{a} w^{2} d x d t \\
& -\frac{s}{2} \int_{Q_{1}}\left(a \varphi_{x x}\right)_{x x} w^{2} d x d t \\
& \left\{\text { B.T.\} } \left\{\begin{array}{l}
-\frac{1}{2} \int_{0}^{j_{1}}\left[w_{x}^{2}\right]_{0}^{T} d x+\int_{0}^{T}\left[w_{x} w_{t}\right]_{0}^{j_{1}} d t \\
+\frac{s}{2} \int_{0}^{T}\left[\left(a \varphi_{x x}\right)_{x} w^{2}\right]_{0}^{j_{1}} d t-s \int_{0}^{T}\left[a \varphi_{x} w_{x}^{2}\right]_{0}^{j_{1}} d t \\
-s \int_{0}^{T}\left[a \varphi_{x x} w w_{x}\right]_{0}^{j_{1}} d t+\frac{1}{2} \int_{0}^{j_{1}}\left[\left(s^{2} \varphi_{x}^{2}-s \frac{\varphi_{t}}{a}\right) w^{2}\right]_{0}^{T} d x \\
-s \int_{0}^{T}\left[\left(s^{2} a \varphi_{x}^{3}-s \varphi_{x} \varphi_{t}\right) w^{2}\right]_{0}^{j_{1}} d t .
\end{array}\right.\right.
\end{aligned}
$$


Proof. It results, integrating by parts,

$$
\begin{aligned}
\int_{Q_{1}} \frac{a w_{x x}}{a} L_{s}^{-} w d x d t= & \int_{Q_{1}} w_{x x} w_{t} d x d t-2 s \int_{Q_{1}} a \varphi_{x} w_{x} w_{x x} d x d t \\
& -s \int_{Q_{1}} a \varphi_{x x} w w_{x x} d x d t \\
= & -\int_{Q_{1}} w_{x t} w_{x} d x d t+\int_{0}^{T}\left[w_{x} w_{t}\right]_{0}^{j_{1}} d t \\
& +s \int_{Q_{1}}\left(w\left(a \varphi_{x x}\right)\right)_{x} w_{x} d x d t-s \int_{0}^{T}\left[a \varphi_{x x} w w_{x}\right]_{0}^{j_{1}} d t \\
& +s \int_{Q_{1}}\left(a \varphi_{x}\right)_{x} w_{x}^{2} d x d t-s \int_{0}^{T}\left[a \varphi_{x} w_{x}^{2}\right]_{0}^{j_{1}} d t \\
& +s \int_{Q_{1}}\left(a \varphi_{x x}\right) w_{x}^{2} d x d t-s \int_{0}^{T}\left[a \varphi_{x x} w w_{x}\right]_{0}^{j_{1}} d t \\
= & -\frac{1}{2} \int_{0}^{j_{1}}\left[w_{x}^{2}\right]_{0}^{T} d x+\int_{0}^{T}\left[w_{x} w_{t}\right]_{0}^{j_{1}} d t \\
& +\frac{1}{2} s \int_{0}^{T}\left[\left(a \varphi_{x x}\right)_{x} w^{2}\right]_{0}^{j_{1}} d t-\frac{1}{2} s \int_{Q_{1}}^{T}\left(a \varphi_{x x}\right)_{x x} w^{2} d x d t \\
& \\
& \\
& \\
& \\
& \\
& \\
&
\end{aligned}
$$

Therefore, integrating again by parts,

$$
\begin{aligned}
& \int_{Q_{1}} \frac{1}{a}\left(-s \varphi_{t} w+s^{2} a(x) \varphi_{x}^{2} w\right) L_{s}^{-} w d x d t \\
& \quad=\int_{Q_{1}}\left(s^{2} \varphi_{x}^{2}-s \frac{\varphi_{t}}{a}\right) w w_{t} d x d t-s \int_{Q_{1}} a \varphi_{x x}\left(s^{2} \varphi_{x}^{2}-s \frac{\varphi_{t}}{a}\right) w^{2} d x d t
\end{aligned}
$$




$$
\begin{aligned}
& -2 s \int_{Q_{1}} a \varphi_{x}\left(s^{2} \varphi_{x}^{2}-s \frac{\varphi_{t}}{a}\right) w w_{x} d x d t \\
& =\frac{1}{2} \int_{Q_{1}}\left(-s^{2} \varphi_{x}^{2}+s \frac{\varphi_{t}}{a}\right)_{t} w^{2} d x d t-s^{3} \int_{Q_{1}} \varphi_{x}^{2}\left(a \varphi_{x x}\right) w^{2} d x d t \\
& +s^{2} \int_{Q_{1}}\left(a \varphi_{x x}\right) \frac{\varphi_{t}}{a} w^{2} d x d t+s \int_{Q_{1}}\left(s^{2} a \varphi_{x}^{3}-s \varphi_{x} \varphi_{t}\right)_{x} w^{2} d x d t \\
& +\frac{1}{2} \int_{0}^{j_{1}}\left[\left(s^{2} \varphi_{x}^{2}-s \frac{\varphi_{t}}{a}\right) w^{2}\right]_{0}^{T} d x-s \int_{0}^{T}\left[\left(s^{2} a \varphi_{x}^{3}-s \varphi_{x} \varphi_{t}\right) w^{2}\right]_{0}^{j_{1}} d t \\
& =\frac{s}{2} \int_{Q_{1}} \frac{\varphi_{t t}}{a} w^{2} d x d t+s^{2} \int_{Q_{1}}\left(\varphi_{t} \varphi_{x x}-\left(\varphi_{x} \varphi_{t}\right)_{x}-\varphi_{x} \varphi_{x t}\right) w^{2} d x d t \\
& +s^{3} \int_{Q_{1}}\left(\left(a \varphi_{x}^{3}\right)_{x}-\varphi_{x}^{2} a \varphi_{x x}\right) w^{2} d x d t+\frac{1}{2} \int_{0}^{j_{1}}\left[\left(s^{2} \varphi_{x}^{2}-s \frac{\varphi_{t}}{a}\right) w^{2}\right]_{0}^{T} d x \\
& +s \int_{0}^{T}\left[\left(s^{2} a \varphi_{x}^{3}-s \varphi_{x} \varphi_{t}\right) w^{2}\right]_{0}^{j_{1}} d t \\
& \left.+\frac{s}{2} \int_{Q_{1}}^{\varphi_{t t}} \frac{1}{a} w_{0}^{2} d x d t-2 s^{2} \int_{Q_{1}}\left[\left(s_{x} \varphi_{x} \varphi_{x t} w^{2} d x d t+s \frac{\varphi_{t}}{a}\right) w^{2}\right]_{0}^{T} d x-s \int_{0}^{T}\left[\left(s^{2} a \varphi_{x}^{3}-s \varphi_{x} \varphi_{t}\right) w^{2}\right]_{0}^{j_{1}} d t .2 a \varphi_{x}^{2} \varphi_{x x}\right) w^{2} d x d t \\
& \quad a_{1}
\end{aligned}
$$

Adding (3.8)-(3.9), (3.7) follows immediately.

The next lemma holds.

LEMMA 3.9. The boundary terms in (3.7) become

$$
\{B . T .\}=-s e^{R j_{1}^{2}} \int_{0}^{T} \theta(t) j_{1} w_{x}^{2}\left(t, j_{1}\right) d t .
$$

Proof. Using the definition of $\varphi$ and the fact that $w\left(t, j_{1}\right)=0$, the boundary terms of $<L_{s}^{+} w, L_{s}^{-} w>_{L_{\frac{1}{a}}^{2}\left(Q_{1}\right)}$ become 


$$
\begin{aligned}
\{B . T .\}= & -\frac{1}{2} \int_{0}^{j_{1}}\left[w_{x}^{2}\right]_{0}^{T} d x+\int_{0}^{T}\left[w_{x} w_{t}\right]_{0}^{j_{1}} d t \\
& +\frac{1}{2} \int_{0}^{j_{1}}\left[\left(s^{2} \theta^{2}\left(\frac{x}{a}\right)^{2} e^{2 R x^{2}}-\frac{s}{a} \dot{\theta}\left(p(x)-2\|p\|_{L^{\infty}\left(J_{1}\right)}\right)\right) w^{2}\right]_{0}^{T} d x \\
& -s \int_{0}^{T} \theta(t)\left[e^{R x^{2}} x w_{x}^{2}\right]_{0}^{j_{1}} d t+\frac{s}{2} \int_{0}^{T} \theta(t)\left[\left(\frac{x a_{x}}{a}\right)_{x} w^{2}\right](t, 0) d t \\
& -s \int_{0}^{T} \theta(t)\left[\left(1-\frac{x a_{x}}{a}\right) w w_{x}\right](t, 0) d t+s^{3} \int_{0}^{T} \theta^{3}(t)\left[\frac{x^{3}}{a^{2}} w^{2}\right](t, 0) d t \\
& +2 s^{2}\|p\|_{L^{\infty}\left(J_{1}\right)} \int_{0}^{T} \theta(t) \dot{\theta}(t)\left[\frac{x}{a} w^{2}\right](t, 0) d t .
\end{aligned}
$$

Since $w \in \mathcal{V}_{1}$, where $\mathcal{V}_{1}$ is as in (3.3), $w \in C^{0}\left([0, T] ; H_{\frac{1}{a}}^{1}\left(J_{1}\right)\right)$. Thus $w_{x}(x, 0), w_{x}(x, T)$ and $\int_{0}^{j_{1}}\left[w_{x}^{2}\right]_{0}^{T} d x$ are well defined and, using the boundary conditions of $w$, it results that

$$
\int_{0}^{j_{1}}\left[w_{x}^{2}\right]_{0}^{T} d x=0
$$

Moreover, since $w \in H^{1}\left(0, T ; H_{\underline{1}}^{1}\left(J_{1}\right)\right), w_{t}(t, 0)$ and $w_{t}\left(t, j_{1}\right)$ are well defined. Now, by Lemma 3.6, we have that $\lim _{x \rightarrow 0} \sqrt{x} w_{x}(t, x)=0$. Since $w_{t x}(t, x) \in L^{2}\left(J_{1}\right)$, then, by Hölder's inequality,

$$
\left|w_{t}(t, x)\right| \leq \int_{0}^{x}\left|w_{t x}(t, y)\right| d y \leq \sqrt{x}\left(\int_{0}^{x}\left|w_{t x}(t, y)\right|^{2} d y\right)^{1 / 2} .
$$

Thus, if $w \in \mathcal{V}_{1}$ then $\int_{0}^{T}\left[w_{x} w_{t}\right]_{0}^{j_{1}} d t$ is well defined and it is 0 . Now, we consider the term

$$
\frac{1}{2} \int_{0}^{j_{1}}\left[\left(s^{2} \theta^{2}\left(\frac{x}{a}\right)^{2} e^{2 R x^{2}}-\frac{s}{a} \dot{\theta}\left(p(x)-2\|p\|_{L^{\infty}\left(J_{1}\right)}\right)\right) w^{2}\right]_{0}^{T} d x
$$


Since $w \in \mathcal{V}_{1}$, then $w \in C^{0}\left([0, T] ; L_{1}^{2}\left(J_{1}\right)\right)$. Thus $w(0, x)$ and $w(T, x)$ are well defined and $w(0, x)=w(T, x)=0$. This implies that

$$
\frac{1}{2} \int_{0}^{j_{1}}\left[\left(s^{2} \theta^{2}\left(\frac{x}{a}\right)^{2} e^{2 R x^{2}}-\frac{s}{a} \dot{\theta}\left(p(x)-2\|p\|_{L^{\infty}\left(J_{1}\right)}\right)\right) w^{2}\right]_{0}^{T} d x=0 .
$$

By Lemma 3.6

$$
-s \int_{0}^{T} \theta(t)\left[e^{R x^{2}} x w_{x}^{2}\right]_{0}^{j_{1}} d t=-s \int_{0}^{T} \theta(t)\left[e^{R x^{2}} x w_{x}^{2}\right]\left(t, j_{1}\right) d t .
$$

Thus, the boundary terms become

$$
\begin{aligned}
\{B . T .\}= & -s e^{R j_{1}^{2}} \int_{0}^{T} \theta(t) j_{1} w_{x}^{2}\left(t, j_{1}\right) d t+\frac{s}{2} \int_{0}^{T} \theta(t)\left[\left(\frac{x a_{x}}{a}\right)_{x} w^{2}\right](t, 0) d t \\
& -s \int_{0}^{T} \theta(t)\left[\left(1-\frac{x a_{x}}{a}\right) w w_{x}\right](t, 0) d t+s^{3} \int_{0}^{T} \theta^{3}(t)\left[\frac{x^{3}}{a^{2}} w^{2}\right](t, 0) d t \\
& +2 s^{2}\|p\|_{L^{\infty}\left(J_{1}\right)} \int_{0}^{T} \theta(t) \dot{\theta}(t)\left[\frac{x}{a} w^{2}\right](t, 0) d t .
\end{aligned}
$$

By Proposition 3.7,

$$
\left|\theta(t)\left[\left(\frac{x a_{x}}{a}\right)_{x} w^{2}\right](t, \epsilon)\right| \leq \theta(t)\left|\epsilon\left(\frac{x a_{x}}{a}\right)_{x}(\epsilon)\right| \int_{0}^{\epsilon} w_{x}^{2}(t, y) d y \rightarrow 0
$$

as $\epsilon \rightarrow 0^{+}$. Thus

$$
\frac{s}{2} \int_{0}^{T} \theta(t)\left[\left(\frac{x a_{x}}{a}\right)_{x} w^{2}\right](t, 0) d t=\lim _{\epsilon \rightarrow 0} \frac{s}{2} \int_{0}^{T} \theta(t)\left[\left(\frac{x a_{x}}{a}\right)_{x} w^{2}\right](t, \epsilon) d t=0 .
$$

Moreover, by assumption, it results

$$
\begin{aligned}
& \left|\theta(t)\left[\left(1-\frac{x a_{x}}{a}\right) w w_{x}\right](t, \epsilon)\right| \\
& \quad \leq \theta(t)\left(1+\left\|\frac{x a_{x}}{a}\right\|_{L^{\infty}\left(J_{1}\right)}\right)\left|w_{x}(\epsilon, t)\right|\left(\epsilon \int_{0}^{\epsilon}\left|w_{x}(t, x)\right|^{2} d x\right)^{\frac{1}{2}} \rightarrow 0
\end{aligned}
$$


as $\epsilon \rightarrow 0^{+}$, thus

$$
-s \int_{0}^{T} \theta(t)\left[\left(1-\frac{x a_{x}}{a}\right) w w_{x}\right](t, 0) d t=-\lim _{\epsilon \rightarrow 0} s \int_{0}^{T} \theta(t)\left[\left(1-\frac{x a_{x}}{a}\right) w w_{x}\right](t, \epsilon) d t=0 .
$$

Now, by Lemma 3.5,

$$
\left|\theta(t) \dot{\theta}(t)\left[\frac{x}{a} w^{2}\right](t, \epsilon)\right| \leq \theta(t)|\dot{\theta}(t)| \frac{\epsilon^{2}}{a(\epsilon)} \int_{0}^{\epsilon} w_{x}^{2}(t, y) d y \rightarrow 0
$$

as $\epsilon \rightarrow 0^{+}$, thus

$$
\begin{aligned}
2 s^{2}\|p\|_{L^{\infty}\left(J_{1}\right)} \int_{0}^{T} \theta(t) \dot{\theta}(t)\left[\frac{x}{a} w^{2}\right](t, 0) d t & =\lim _{\epsilon \rightarrow 0} 2 s^{2}\|p\|_{L^{\infty}\left(J_{1}\right)} \int_{0}^{T} \theta(t) \dot{\theta}(t)\left[\frac{x}{a} w^{2}\right](t, \epsilon) d t \\
& =0 .
\end{aligned}
$$

Finally,

$$
s^{3} \int_{0}^{T} \theta^{3}(t)\left[\frac{x^{3}}{a^{2}} w^{2}\right](t, 0) d t=\lim _{\epsilon \rightarrow 0} s^{3} \int_{0}^{T} \theta^{3}(t)\left[\frac{x^{3}}{a^{2}} w^{2}\right](t, \epsilon) d t=0 .
$$

In fact, by Hölder's inequality, it results $w^{2}(t, x) \leq x \int_{0}^{x} w_{x}^{2}(t, y) d y$. Thus

$$
\int_{0}^{T} \theta^{3}(t)\left[\frac{x^{3}}{a^{2}} w^{2}\right](t, \epsilon) d t \leq \int_{0}^{T} \theta^{3}(t)\left[\frac{x^{4}}{a^{2}} \int_{0}^{x} w_{x}^{2} d y\right](t, \epsilon) d t
$$

and again, by Lemma 3.5,

$$
\int_{0}^{T} \theta^{3}(t)\left[\frac{x^{4}}{a^{2}} \int_{0}^{x} w_{x}^{2} d y\right](t, \epsilon) d t \rightarrow 0, \quad \text { as } \quad \epsilon \rightarrow 0
$$

The crucial step is to prove now the following estimate. 
LEMMA 3.10. The distributed terms of (3.7) satisfy the following estimate

$$
\begin{aligned}
& s\left(2-K_{1}\right) \int_{Q_{1}} \theta w_{x}^{2} d x d t+s^{3}\left(2-K_{1}\right) \int_{Q_{1}} \theta^{3}\left(\frac{x}{a}\right)^{2} w^{2} d x d t \\
& -2 s^{2} C \int_{Q_{1}} \theta^{3}\left(\frac{x}{a}\right)^{2} w^{2} d x d t-s C \int_{Q_{1}} \frac{\theta^{\frac{3}{2}}}{a} w^{2} d x d t \\
& \leq s \int_{Q_{1}}\left(a \varphi_{x x}+\left(a \varphi_{x}\right)_{x}\right) w_{x}^{2} d x d t+s^{3} \int_{Q_{1}} \varphi_{x}^{2}\left(a \varphi_{x x}+\left(a \varphi_{x}\right)_{x}\right) w^{2} d x d t \\
& -2 s^{2} \int_{Q_{1}} \varphi_{x} \varphi_{x t} w^{2} d x d t+\frac{s}{2} \int_{Q_{1}} \frac{\varphi_{t t}}{a} w^{2} d x d t-\frac{s}{2} \int_{Q_{1}}\left(a \varphi_{x x}\right)_{x x} w^{2} d x d t,
\end{aligned}
$$

for some positive constant $C$.

Proof. The distributed terms of $\left\langle L_{s}^{+} w, L_{s}^{-} w>_{L_{\frac{1}{a}}^{2}\left(Q_{1}\right)}\right.$, using the definition of $\varphi$, take the form

$$
\begin{aligned}
\{D . T .\}= & s \int_{Q_{1}} \theta\left(2-\frac{x a_{x}}{a}+4 R x^{2}\right) e^{R x^{2}} w_{x}^{2} d x d t \\
& +s^{3} \int_{Q_{1}} \theta^{3}\left(\frac{x}{a}\right)^{2}\left(2-\frac{x a_{x}}{a}+4 R x^{2}\right) e^{3 R x^{2}} w^{2} d x d t \\
& -2 s^{2} \int_{Q_{1}} \theta \dot{\theta}\left(\frac{x}{a}\right)^{2} e^{2 R x^{2}} w^{2} d x d t+\frac{s}{2} \int_{Q_{1}} \frac{\ddot{\theta}}{a}\left(p-2\|p\|_{L^{\infty}\left(J_{1}\right)}\right) w^{2} d x d t \\
& +\frac{s}{2} \int_{Q_{1}} \theta\left(\frac{x a_{x}}{a}\right)_{x x} e^{R x^{2}} w^{2} d x d t+2 s \int_{Q_{1}} \theta R x\left(\frac{x a_{x}}{a}\right)_{x} e^{R x^{2}} w^{2} d x d t \\
& +s \int_{Q_{1}} \theta R\left(\left(1+2 R x^{2}\right)\left(\frac{x a_{x}}{a}\right)-\left(3+12 R x^{2}+4 R^{2} x^{4}\right)\right) e^{R x^{2}} w^{2} d x d t .
\end{aligned}
$$

Because of Hypothesis 3.1.1

$$
2-\frac{x a_{x}}{a} \geq 2-K_{1}>0 \quad \forall x \in(0, \varepsilon) ;
$$

thus there exists $R>0$ such that

$$
2-\frac{x a_{x}}{a}+4 R x^{2} \geq 2-K_{1} \quad \forall x \in J_{1} .
$$


Moreover, using again Hypothesis 3.1.1 and Proposition 3.7 for all $x \in J_{1}$ one has

$$
\begin{aligned}
& R\left|\left(2 x\left(\frac{x a_{x}}{a}\right)_{x}+\left(1+2 R x^{2}\right)\left(\frac{x a_{x}}{a}\right)-\left(3+12 R x^{2}+4 R^{2} x^{4}\right)\right)\right| \\
& \quad \leq R\left(2\left\|x\left(\frac{x a_{x}}{a}\right)_{x}\right\|_{L^{\infty}\left(J_{1}\right)}+(1+2 R)\left\|\left(\frac{x a_{x}}{a}\right)\right\|_{L^{\infty}\left(J_{1}\right)}+\left(3+12 R+4 R^{2}\right)\right)=: C_{R} .
\end{aligned}
$$

Then, seth $C_{1}^{\prime}:=C_{1} \vee \max _{\left[\varepsilon, j_{1}\right]}\left|\left(\frac{x a_{x}}{a}\right)_{x x}\right|$, where $C_{1}$ is the constant of Hypothesis $3.1_{\bar{\kappa}}$ one has

$$
\begin{aligned}
\{D . T .\} \geq & s\left(2-K_{1}\right) \int_{Q_{1}} \theta w_{x}^{2} d x d t+s^{3}\left(2-K_{1}\right) \int_{Q_{1}} \theta^{3}\left(\frac{x}{a}\right)^{2} w^{2} d x d t \\
& -2 s^{2} e^{2 R} \int_{Q_{1}} \theta|\dot{\theta}|\left(\frac{x}{a}\right)^{2} w^{2} d x d t-s\|p\|_{L^{\infty}\left(J_{1}\right)} \int_{Q_{1}} \frac{|\ddot{\theta}|}{a} w^{2} d x d t \\
& -s^{R}\left(C_{1}^{\prime}+C_{R}\right)\left(1 \vee\|a\|_{L^{\infty}(0,1)}\right) \int_{Q_{1}} \frac{\theta}{a} w^{2} d x d t .
\end{aligned}
$$

Observing that there exists $C_{T}>0$ such that $\theta|\dot{\theta}| \leq C_{T} \theta^{3},|\ddot{\theta}| \leq C_{T} \theta^{\frac{3}{2}}$ and $\theta \leq C_{T} \theta^{\frac{3}{2}}$, one can deduce the next estimate:

$$
\begin{aligned}
\{D . T .\} \geq & s\left(2-K_{1}\right) \int_{Q_{1}} \theta w_{x}^{2} d x d t+s^{3}\left(2-K_{1}\right) \int_{Q_{1}} \theta^{3}\left(\frac{x}{a}\right)^{2} w^{2} d x d t \\
& -2 s^{2} e^{2 R} C_{T} \int_{Q_{1}} \theta^{3}\left(\frac{x}{a}\right)^{2} w^{2} d x d t \\
& -s e^{R} C_{T}\left(\left(C_{1}^{\prime}+C_{R}\right)\left(1 \vee\|a\|_{L^{\infty}(0,1)}\right)+\|p\|_{L^{\infty}\left(J_{1}\right)}\right) \int_{Q_{1}} \frac{\theta^{\frac{3}{2}}}{a} w^{2} d x d t .
\end{aligned}
$$

PROPOSITION 3.11. There exist two positive constants $C$ and $s_{0}$ such that, for all $s \geq s_{0}$, all solutions $w$ of (3.5) in $\mathcal{V}_{1}$ satisfy

$$
\int_{Q_{1}} s \theta w_{x}^{2}+s^{3} \theta^{3}\left(\frac{x}{a}\right)^{2} w^{2} d x d t \leq C\left(\int_{Q_{1}} h^{2} \frac{e^{2 s \varphi}}{a} d x d t+2 s \int_{0}^{T} \theta(t) j_{1} w_{x}^{2}\left(t, j_{1}\right) d t\right) .
$$


Proof. By (3.6) and by Lemmas 3.9 and 3.10 it is sufficient to estimate only the term $-s C \int_{Q_{1}} \frac{\theta^{\frac{3}{2}}}{a} w^{2} d x d t$. For $\lambda>0$ it results

$$
\begin{aligned}
\int_{Q_{1}} \frac{\theta^{\frac{3}{2}}}{a} w^{2} d x d t & =\int_{Q_{1}}\left(\frac{1}{\lambda} \theta^{2}\left(\frac{x}{a}\right)^{2} w^{2}\right)^{\frac{1}{2}}\left(\lambda \frac{\theta}{x^{2}} w^{2}\right)^{\frac{1}{2}} d x d t \\
& \leq \frac{1}{\lambda} \int_{Q_{1}} \theta^{2}\left(\frac{x}{a}\right)^{2} w^{2} d x d t+\lambda \int_{Q_{1}} \frac{\theta}{x^{2}} w^{2} d x d t
\end{aligned}
$$

By Hardy's inequality one has

$$
\int_{Q_{1}} \frac{\theta^{\frac{3}{2}}}{a} w^{2} d x d t \leq \frac{1}{\lambda} \int_{Q_{1}} \theta^{2}\left(\frac{x}{a}\right)^{2} w^{2} d x d t+\lambda C_{H} \int_{Q_{1}} \theta w_{x}^{2} d x d t
$$

for some positive constant $C_{H}$. Thus, for $s_{0}$ large enough and $\lambda$ small enough,

$$
\begin{aligned}
& C_{\lambda}\left(\int_{Q_{1}} \theta w_{x}^{2} d x d t+s^{3} \int_{Q_{1}} \theta^{3}\left(\frac{x}{a}\right)^{2} w^{2} d x d t\right)-2 s e^{R j_{1}^{2}} \int_{0}^{T} \theta(t) j_{1} w_{x}^{2}\left(t, j_{1}\right) d t \\
& \quad \leq \int_{Q_{1}} h^{2} \frac{e^{2 s \varphi}}{a} d x d t,
\end{aligned}
$$

for some positive constant $C_{\lambda}$ and for all $s \geq s_{0}$.

Recalling the definition of $w$, we have $v=e^{-s \varphi} w$ and $v_{x}=\left(w_{x}-s \varphi_{x} w\right) e^{-s \varphi}$. Thus, Theorem 3.3 follows immediately by Proposition 3.11 .

\section{Observability and controllability of linear equations}

In this section we will prove, as a consequence of the Carleman estimates established in Section 3, an observability inequality for the adjoint problem

$$
\begin{cases}v_{t}+a(x) v_{x x}=0, & (t, x) \in Q, \\ v(t, 0)=v(t, 1)=0, & t \in(0, T), \\ v(T, x)=v_{T}(x) \in L_{\frac{1}{a}}^{2}(0,1) & \end{cases}
$$

of (2.1). In particular, the following result holds. 
PROPOSITION 4.1. Assume that Hypothesis 3.1 is satisfied. Then there exists a positive constant $C_{T}$ such that every solution $v \in \mathcal{U}$ of (4.1) satisfies

$$
\int_{0}^{1} v^{2}(0, x) \frac{1}{a} d x \leq C_{T} \int_{0}^{T} \int_{\omega} v^{2} \frac{1}{a} d x d t .
$$

Here $\mathcal{U}:=C^{0}\left([0, T] ; L_{\frac{1}{a}}^{2}(0,1)\right) \cap L^{2}\left(0, T ; H_{\frac{1}{a}}^{1}(0,1)\right)$.

Before proving this proposition we will give some results that will be very helpful to this aim. As a first step we introduce the following class of functions

$$
\mathcal{W}:=\left\{v \text { solution of }(4.1) \mid v_{T} \in D\left(A^{2}\right)\right\},
$$

where

$$
D\left(A^{2}\right)=\left\{u \in H_{\frac{1}{a}}^{1}(0,1) \mid a u_{x x} \in H_{\frac{1}{a}}^{2}(0,1)\right\} .
$$

Obviously,

$$
\begin{aligned}
\mathcal{W} & \subset C^{1}\left([0, T] ; H_{\frac{1}{a}}^{2}(0,1)\right) \\
& \subset \mathcal{V}:=L^{2}\left(0, T ; H_{\frac{1}{a}}^{2}(0,1)\right) \cap H^{1}\left(0, T ; H_{\frac{1}{a}}^{1}(0,1)\right) \subset \mathcal{U}
\end{aligned}
$$

PROPOSITION 4.2. (Caccioppoli's inequality) Let $\omega^{\prime}$ and $\omega$ two open subintervals of $(0,1)$ such that $\omega^{\prime} \subset \subset \omega \subset \subset(0,1)$. Let $s>0$ and $\psi(t, x):=\theta(t) \Psi(x)$, where $\theta$ is defined in (3.2) and $\Psi \in C^{1}(0,1)$ is a strictly negative function. Then, there exists a positive constant $C$ such that

$$
\int_{0}^{T} \int_{\omega^{\prime}} v_{x}^{2} e^{2 s \psi} d x d t \leq C \int_{0}^{T} \int_{\omega} v^{2} d x d t
$$

for every solution $v$ of the adjoint problem (4.1).

Proof. Let us consider a smooth function $\xi:[0,1] \rightarrow \mathbb{R}$ such that

$$
\begin{cases}0 \leq \xi(x) \leq 1, & \text { for all } x \in[0,1] \\ \xi(x)=1, & x \in \omega^{\prime}, \\ \xi(x)=0, & x \in(0,1) \backslash \omega .\end{cases}
$$


Then,

$$
\begin{aligned}
0 & =\int_{0}^{T} \frac{d}{d t}\left(\int_{0}^{1}\left(\xi e^{s \psi}\right)^{2} v^{2} d x\right) d t=\int_{Q} 2 s \psi_{t}\left(\xi e^{s \psi}\right)^{2} v^{2}+2\left(\xi e^{s \psi}\right)^{2} v\left(-a v_{x x}\right) d x d t \\
& =2 s \int_{Q} \psi_{t}\left(\xi e^{s \psi}\right)^{2} v^{2} d x d t+2 \int_{Q}\left(\xi^{2} e^{2 s \psi} a\right)_{x} v v_{x} d x d t+2 \int_{Q}\left(\xi^{2} e^{2 s \psi} a\right) v_{x}^{2} d x d t
\end{aligned}
$$

Hence,

$$
\begin{aligned}
2 \int_{Q}\left(\xi^{2} e^{2 s \psi} a\right) v_{x}^{2} d x d t= & -2 s \int_{Q} \psi_{t}\left(\xi e^{s \psi}\right)^{2} v^{2} d x d t \\
& -2 \int_{Q}\left(\xi^{2} e^{2 s \psi} a\right)_{x} \frac{\xi e^{s \psi} \sqrt{a}}{\xi e^{s \psi} \sqrt{a}} v v_{x} d x d t \\
\leq & -2 s \int_{Q} \psi_{t}\left(\xi e^{s \psi}\right)^{2} v^{2} d x d t+4 \int_{Q}\left(\xi e^{s \psi} \sqrt{a}\right)_{x}^{2} v^{2} d x d t \\
& +\int_{Q}\left(\xi^{2} e^{2 s \psi} a\right) v_{x}^{2} d x d t .
\end{aligned}
$$

Thus,

$$
\inf _{\omega^{\prime}}\{a\} \int_{0}^{T} \int_{\omega^{\prime}} e^{2 s \psi} v_{x}^{2} d x d t \leq \sup _{\omega \times(0, T)}\left\{\left|4\left(\xi e^{s \psi} \sqrt{a}\right)_{x}^{2}-2 s \psi_{t}\left(\xi e^{s \psi}\right)^{2}\right|\right\} \int_{0}^{T} \int_{\omega} v^{2} d x d t .
$$

As a consequence of Proposition 4.2 one has:

LEMMA 4.3. Assume that Hypothesis 3.1 is satisfied. Let $T_{0}, T_{1}$ be such that $0<$ $T_{0}<T_{1}<T$. Then there exists a positive constant $C=C\left(T_{0}, T_{1}\right)$ such that every solution $v \in \mathcal{W}$ of (4.1) satisfies

$$
\int_{T_{0}}^{T_{1}} \int_{0}^{1} v^{2} \frac{1}{a} d x d t \leq C \int_{0}^{T} \int_{\omega} v^{2} \frac{1}{a} d x d t
$$


Proof. Let us consider a smooth function $\xi:[0,1] \rightarrow \mathbb{R}$ such that

$$
\begin{cases}0 \leq \xi(x) \leq 1, & \text { for all } x \in[0,1], \\ \xi(x)=1, & x \in[0,(2 \alpha+\beta) / 3], \\ \xi(x)=0, & x \in[(\alpha+2 \beta) / 3,1] .\end{cases}
$$

We define $w(t, x):=\xi(x) v(t, x)$ where $v \in \mathcal{W}$. Then $w$ satisfies

$$
\begin{cases}w_{t}+a w_{x x}=a\left(\xi_{x x} v+2 \xi_{x} v_{x}\right)=: h, & (t, x) \in(0, T) \times(0, \beta), \\ w(t, 0)=w(t, \beta)=0, & t \in(0, T) .\end{cases}
$$

Setting $w^{\prime}:=((2 \alpha+\beta) / 3,(\alpha+2 \beta) / 3)$ and using Proposition 4.2, it results

$$
\begin{aligned}
\int_{Q} h^{2} \frac{e^{2 s \psi}}{a} d x d t & =\int_{0}^{T} \int_{w^{\prime}} a\left(\xi_{x x} v+2 \xi_{x} v_{x}\right)^{2} e^{2 s \psi} d x d t \\
& \leq C \int_{0}^{T} \int_{w^{\prime}} a\left(\xi_{x x}^{2} v^{2}+4 \xi_{x}^{2} v_{x}^{2}\right) e^{2 s \psi} d x d t \\
& \leq C \int_{0}^{T} \int_{w^{\prime}} v^{2} d x d t+C \int_{0}^{T} \int_{w^{\prime}} v_{x}^{2} e^{2 s \psi} d x d t \\
& \leq C \int_{0}^{T} \int_{w} v^{2} d x d t \leq C \int_{0} \int_{w} v^{2} \frac{1}{a} d x d t
\end{aligned}
$$

for some positive constant $C$. Applying the previous inequality with $\psi=\varphi_{1}$ and Theorem 3.3 with $J_{1}=(0, \beta)$, one has

$$
\begin{aligned}
\int_{0}^{T} \int_{\omega} v^{2} \frac{1}{a} d x d t & \geq C \int_{Q} h^{2} \frac{e^{2 s_{0} \varphi_{1}}}{a} d x d t \geq C \int_{0}^{T} \int_{0}^{\beta} s_{0} \theta w_{x}^{2} e^{2 s_{0} \varphi_{1}} d x d t \\
& \geq C \int_{T_{0}}^{T_{1}} \int_{0}^{\beta} w_{x}^{2} d x d t .
\end{aligned}
$$

By Proposition 2.6 it follows

$$
\int_{0}^{T} \int_{\omega} v^{2} \frac{1}{a} d x d t \geq C \int_{T_{0}}^{T_{1}} \int_{0}^{\beta} w^{2} \frac{1}{a} d x d t \geq C \int_{T_{0}}^{T_{1}} \int_{0}^{(2 \alpha+\beta) / 3} v^{2} \frac{1}{a} d x d t .
$$


Consider now $z(t, x):=(1-\xi(x)) v(t, x)$. Then $z$ satisfies

$$
\begin{cases}z_{t}+a z_{x x}=-h, & (t, x) \in(0, T) \times(\alpha, 1), \\ z(t, \alpha)=z(t, 1)=0, & t \in(0, T),\end{cases}
$$

and, as before, using (4.4) with $\psi=\varphi_{2}$, Theorem 3.4 with $J_{2}=(\alpha, 1)$ and Proposition 2.6, it results

$$
\int_{0}^{T} \int_{\omega} v^{2} \frac{1}{a} d x d t \geq C \int_{T_{0}}^{T_{1}} \int_{(\alpha+2 \beta) / 3}^{1} v^{2} \frac{1}{a} d x d t .
$$

By (4.5) and (4.6), one has

$$
\int_{0}^{T} \int_{w} v^{2} \frac{1}{a} d x d t \geq C \int_{T_{0}}^{T_{1}} \int_{w^{c}}^{1} v^{2} \frac{1}{a} d x d t
$$

where $w^{c}:=(0,1) \backslash w$. Thus

$$
\begin{aligned}
2 \int_{0}^{T} \int_{w} v^{2} \frac{1}{a} d x d t & \geq \int_{0}^{T} \int_{w} v^{2} \frac{1}{a} d x d t+\int_{T_{0}}^{T_{1}} \int_{w} v^{2} \frac{1}{a} d x d t \\
& \geq C \int_{T_{0}}^{T_{1}} \int_{w^{c}} v^{2} \frac{1}{a} d x d t+\int_{T_{0}}^{T_{1}} \int_{w} v^{2} \frac{1}{a} d x d t \\
& \geq(1 \wedge C) \int_{T_{0}}^{T_{1}} \int_{0}^{1} v^{2} \frac{1}{a} d x d t
\end{aligned}
$$

for some positive constant $C$.

LEMMA 4.4. Assume that Hypothesis 3.1 is satisfied. Let $T_{0}, T_{1}$ be such that $0<T_{0}<$ $T_{1}<T$. Then every solution $v \in \mathcal{W}$ of (4.1) satisfies,

$$
\int_{0}^{1} v^{2}(0, x) \frac{1}{a} d x \leq \frac{1}{T_{1}-T_{0}} \int_{T_{0}}^{T_{1}} \int_{0}^{1} v^{2} \frac{1}{a} d x d t .
$$


Proof. Multiplying (4.1) by $\frac{v}{a}$ and integrating over $(0,1)$, one has

$$
\begin{aligned}
0 & =\int_{0}^{1} v_{t}(t, x) v(t, x) \frac{1}{a} d x+\int_{0}^{1} v_{x x}(t, x) v(t, x) d x \\
& =\frac{1}{2} \frac{d}{d t} \int_{0}^{1} v^{2}(t, x) \frac{1}{a} d x-\int_{0}^{1} v_{x}^{2}(t, x) d x .
\end{aligned}
$$

Then

$$
\frac{d}{d t} \int_{0}^{1} v^{2}(t, x) \frac{1}{a} d x=2 \int_{0}^{1} v_{x}^{2}(t, x) d x \geq 0 \quad \forall t \in[0, T]
$$

that is the function $t \mapsto \int_{0}^{1} v^{2}(t, x) \frac{1}{a} d x$ is nondecreasing for all $t \in[0, T]$.

Proof of Proposition 4.1. As a direct consequence of the Lemmas 4.3 and 4.4 we have that the observability inequality (4.2) hold for all $v \in \mathcal{W}$. Now, let $v_{T} \in L_{\frac{1}{a}}^{2}(0,1)$ and $v$ the solution of (4.1) associated to $v_{T}$. Since $D\left(A^{2}\right)$ is densely contained in $L_{\frac{1}{a}}^{2}(0,1)$, there exists a sequence $\left(v_{T}^{n}\right)_{n} \subset D\left(A^{2}\right)$ which converges to $v_{T}$ in $L_{\frac{1}{a}}^{2}(0,1)$. Consider now the solution $v_{n}$ associated to $v_{T}^{n}$. Obviously, $\left(v_{n}\right)_{n}$ converges to $v$ in $L^{\infty}\left(0, T ; L_{\frac{1}{a}}^{2}(0,1)\right) \cap$ $L^{2}\left(0, T ; H_{\frac{1}{a}}^{1}(0,1)\right)($ see, e.g., $[2])$ and

$$
\int_{0}^{1} v_{n}^{2}(0, x) \frac{1}{a} d x \leq C_{T} \int_{0}^{T} \int_{\omega} v_{n}^{2} \frac{1}{a} d x d t
$$

Clearly,

$$
\lim _{n \rightarrow+\infty} \int_{0}^{T} \int_{\omega} v_{n}^{2} \frac{1}{a} d x d t=\int_{0}^{T} \int_{\omega} v^{2} \frac{1}{a} d x d t
$$

and

$$
\lim _{n \rightarrow+\infty} \int_{0}^{1} v_{n}^{2}(0, x) \frac{1}{a} d x=\int_{0}^{1} v^{2}(0, x) \frac{1}{a} d x
$$


Assuming that Hypothesis 3.1 is satisfied, using the observability property proved in Proposition 4.1 and a standard technique, one can prove a null controllability result for the linear degenerate problem (2.1):

THEOREM 4.5. Assume that Hypotheses 3.1 is satisfied. Then, given $T>0$ and $u_{0} \in$ $L_{\frac{1}{a}}^{2}(0,1)$, there exists $f \in L^{2}(Q)$ such that the solution $u$ of (2.1) satisfies

$$
u(T, x)=0 \text { for every } x \in[0,1] .
$$

Moreover

$$
\int_{Q} \chi_{\omega} f^{2} \frac{1}{a} d x d t \leq C \int_{0}^{1} u_{0}^{2} \frac{1}{a} d x
$$

for some positive constant $C$.

REMARK 4.6. We observe that if $K_{1}$ or $K_{2}>2$, then problem (2.1) fails to be null controllable. To see this, it is sufficient to consider a coefficient $a$ which degenerates only at zero. For example, we can take $a(x)=x^{K}$ with $K>2$. Using the standard change of variables

$$
X:=\int_{x}^{1} \frac{d y}{\sqrt{a(y)}}, \quad U(t, X):=a(x)^{-1 / 4} u(t, x),
$$

the degenerate equation

$$
u_{t}-a(x) u_{x x}=f \chi_{\omega}
$$

set in $(0,1)$ with $\omega \subset \subset(0,1)$ becomes

$$
U_{t}-U_{X X}+b(X) U=F \chi_{\tilde{\omega}},
$$

now set in $(0,+\infty)$, where $\tilde{\omega} \subset \subset(0,+\infty)$ and $b(X)$ is given by

$$
b(X)=\frac{K}{4}\left(\frac{3 K}{4}-1\right)\left(1+\left(\frac{K}{2}-1\right) X\right)^{-2} .
$$

Thus the degerate heat equation in $(0,1)$ is traslate in a non degenerate heat equation in the unbounded domain $(0, \infty)$ with a regular potential term. Using a result of Escauriaza, Seregin and Sverak (see [12] or [13]), that generalizes previous work by Micu and Zuazua (see [26] or [27]), we deduce, as in [7], that (4.7) is not null controllable. With an analogous technique and more sophisticated calculations, one can obtain the same conclusion also when $K=2$. 


\section{Linear and semilinear extensions}

\subsection{A linear extension.}

In this subsection, we will extend the results established in the previous sections to the following degenerate parabolic problem

$$
\begin{cases}u_{t}-a(x) u_{x x}+b(t, x) u=f(t, x) \chi_{\omega}(x), & (t, x) \in Q, \\ u(t, 0)=u(t, 1)=0, & t \in(0, T), \\ u(0, x)=u_{0}(x), & x \in(0,1),\end{cases}
$$

where, as before, $Q:=(0, T) \times(0,1), T>0$ is fixed, $\omega:=(\alpha, \beta) \subset \subset(0,1)$ is a non-empty assigned interval, $\left(f, u_{0}\right) \in L^{2}(Q) \times L_{\frac{1}{a}}^{2}(0,1)$ and $a \in C^{0}[0,1]$ is such that $a(0)=a(1)=0$ and $a>0$ on $(0,1)$. Furthermore we assume that the potential $b=b(t, x)$ is essentially bounded on $Q$.

Using a perturbation argument, one can prove that Theorem 2.4 still hold for (5.1), that is (5.1) is well-posed in the sense of semigroup theory:

THEOREM 5.1. For all $f \in L^{2}(Q)$ and $u_{0} \in L_{\frac{1}{a}}^{2}(0,1)$, there exists a unique weak solution $u \in \mathcal{U}:=C^{0}\left([0, T] ; L_{\frac{1}{a}}^{2}(0,1)\right) \cap L^{2}\left(0, T ; H_{\frac{1}{a}}^{1}(0,1)\right)$ of (5.1). Moreover, one has

$$
\sup _{t \in[0, T]}\|u(t)\|_{L_{\frac{1}{a}}^{2}(0,1)}^{2}+\int_{0}^{T}\|u\|_{H_{\frac{1}{a}}^{1}(0,1)}^{2} d t \leq C\left(\left\|u_{0}\right\|_{L_{\frac{1}{a}}^{2}(0,1)}^{2}+\int_{0}^{T}\|f\|_{L_{\frac{1}{a}}^{2}(\omega)}^{2} d t\right),
$$

for thpositive constant $C$.

Now, we have to prove that the observability property and the null controllability result obtained in Section 4 still hold for the adjoint problem of (5.1). To this purpose first we have to establish $h_{\lambda}$ for

$$
\begin{cases}v_{t}+a(x) v_{x x}-b(x, t) v=h(t, x), & (t, x) \in Q_{i}:=(0, T) \times J_{i}, \\ v\left(t, \partial J_{i}\right)=0, & t \in(0, T)_{\wedge}\end{cases}
$$

Carleman estimates similar to the ones proved in Theorems 3.3 and 3.4. Here, as in Section $3, T>0$ is fixed, $J_{1}:=\left(0, j_{1}\right)$ and $J_{2}:=\left(j_{2}, 1\right)$ are proper subintervals of $(0,1)$ and $h \in L^{2}\left(0, T ; L_{\frac{1}{a}}^{2}(0,1)\right)$. Then, one has the following: 
PROPOSITION 5.2. Assume that the potential $b \in L^{\infty}(Q)$ and that Hypothesis 3.1 holds for some $\varepsilon \in(0,1)$ such that $\varepsilon<j_{1}$ and $1-\varepsilon>j_{2}$. Then, there exist two positive constants $C$ and $s_{0}$, such that, for all $s \geq s_{0}$, the following Carleman estimates hold

$$
\begin{aligned}
\int_{Q_{1}}\left(s \theta v_{x}^{2}+s^{3} \theta^{3}\left(\frac{x}{a}\right)^{2} v^{2}\right) e^{2 s \varphi_{1}} d x d t \leq & C \int_{Q_{1}} h^{2} \frac{e^{2 s \varphi_{1}}}{a} d x d t \\
& +s C \int_{0}^{T} \theta(t)\left[x v_{x}^{2} e^{2 s \varphi_{1}}\right]\left(t, j_{1}\right) d t,
\end{aligned}
$$

for all solution $v \in \mathcal{V}_{1}$ of (5.2) and

$$
\begin{aligned}
\int_{Q_{2}}\left(s \theta v_{x}^{2}+s^{3} \theta^{3}\left(\frac{x-1}{a}\right)^{2} v^{2}\right) e^{2 s \varphi_{2}} d x d t \leq & C \int_{Q_{2}} h^{2} \frac{e^{2 s \varphi_{2}}}{a} d x d t \\
& +s C \int_{0}^{T} \theta(t)\left[(1-x) v_{x}^{2} e^{2 s \varphi_{2}}\right]\left(t, j_{2}\right) d t,
\end{aligned}
$$

for all solution $v \in \mathcal{V}_{2}$ of (5.2).

Proof. We will prove only the first estimate since the proof of the second one is analogous. Rewrite the equation of (5.2) as $v_{t}+a v_{x x}=\bar{h}$, where $\bar{h}:=h+b v$. Then, as a consequence of Theorem 3.3, there exist two positive constants $C$ and $s_{0}>0$, such that, for all $s \geq s_{0}$,

$$
\begin{aligned}
\int_{Q_{1}}\left(s \theta v_{x}^{2}+s^{3} \theta^{3}\left(\frac{x}{a}\right)^{2} v^{2}\right) e^{2 s \varphi_{1}} d x d t \leq & C \int_{Q_{1}}|\bar{h}|^{2} \frac{e^{2 s \varphi_{1}}}{a} d x d t \\
& +s C \int_{0}^{T} \theta(t)\left[x v_{x}^{2} e^{2 s \varphi_{1}}\right]\left(t, j_{1}\right) d t .
\end{aligned}
$$

By the definition of $\bar{h}$ the term $\int_{Q_{1}}|\bar{h}|^{2} \frac{e^{2 s \varphi_{1}}}{a} d x d t$ can be estimated in the following way

$$
\int_{Q_{1}}|\bar{h}|^{2} \frac{e^{2 s \varphi_{1}}}{a} d x d t \leq 2 \int_{Q_{1}}\left(|h|^{2}+|b|^{2} v^{2}\right) \frac{e^{2 s \varphi_{1}}}{a} d x d t .
$$

But, as a consequence of Proposition 2.6,

$$
\begin{aligned}
\int_{Q_{1}}|b|^{2} v^{2} \frac{e^{2 s \varphi_{1}}}{a} d x d t & \leq\|b\|_{\infty}^{2} \int_{Q_{1}}\left(e^{s \varphi_{1}} v\right)^{2} \frac{1}{a} d x d t \leq\|b\|_{\infty}^{2} \int_{Q_{1}}\left(e^{s \varphi_{1}} v\right)_{x}^{2} d x d t \\
& \leq C \int_{Q_{1}} e^{2 s \varphi_{1}} v_{x}^{2} d x d t+C s^{2} \int_{Q_{1}} \theta^{2} e^{2 s \varphi_{1}}\left(\frac{x}{a}\right)^{2} v^{2} d x d t
\end{aligned}
$$


Using this last inequality in (5.4), it follows

$$
\begin{aligned}
\int_{Q_{1}}|\bar{h}|^{2} \frac{e^{2 s \varphi_{1}}}{a} d x d t \leq & 2 \int_{Q_{1}}|h|^{2} \frac{e^{2 s \varphi_{1}}}{a} d x d t+C \int_{Q_{1}} e^{2 s \varphi_{1}} v_{x}^{2} d x d t \\
& +C s^{2} \int_{Q_{1}} \theta^{2} e^{2 s \varphi_{1}}\left(\frac{x}{a}\right)^{2} v^{2} d x d t .
\end{aligned}
$$

Substituting in (5.3), one can conclude

$$
\begin{aligned}
& \int_{Q_{1}}\left(s \theta v_{x}^{2}+s^{3} \theta^{3}\left(\frac{x}{a}\right)^{2} v^{2}\right) e^{2 s \varphi_{1}} d x d t \leq C\left(\int_{Q_{1}}|h|^{2} \frac{e^{2 s \varphi_{1}}}{a} d x d t\right. \\
& \left.+\int_{Q_{1}} e^{2 s \varphi_{1}} v_{x}^{2} d x d t+s^{2} \int_{Q_{1}} \theta^{2} e^{2 s \varphi_{1}}\left(\frac{x}{a}\right)^{2} v^{2} d x d t+s \int_{0}^{T} \theta(t)\left[x v_{x}^{2} e^{2 s \varphi_{1}}\right]\left(t, j_{1}\right) d t\right) .
\end{aligned}
$$

Hence, for all $s \geq s_{0}$, where $s_{0}$ is assumed sufficiently large, the first estimate of Proposition 5.2 is proved.

As a consequence of the previous Carleman estimates, one can deduce an observability inequality for the adjoint problem

$$
\begin{cases}v_{t}+a(x) v_{x x}-b(t, x) v=0, & (t, x) \in Q, \\ v(t, 0)=v(t, 1)=0, & t \in(0, T), \\ v(T)=v_{T} \in L_{\frac{1}{a}}^{2}(0,1) & \end{cases}
$$

of (5.1). Without loss of generality we can assume that $b \geq 0$. (Otherwise one can reduce the problem to this case introducing $\tilde{u}:=e^{-\lambda t} u$ for a suitable $\lambda>0$.) Moreover, we observe that in a way analogous the proof of Proposition 4.2, it is possible to prove that the Caccioppoli's inequality (4.3) is satisfies for all solution of (5.6).

PROPOSITION 5.3. Assume that the potential $b \in L^{\infty}(Q)$ and that Hypothesis 3.1 is satisfied. Then, there exists a positive constant $C_{T}$ such that every solution $v \in \mathcal{U}$ of (5.6) satisfies

$$
\int_{0}^{1} v^{2}(0, x) \frac{1}{a} d x \leq C_{T} \int_{0}^{T} \int_{\omega} v^{2} \frac{1}{a} d x d t .
$$


Proof. As in the proof of Lemma 4.4 and using the fact that $b \geq 0$, it results that every $v \in \mathcal{W}^{\prime}:=\left\{v\right.$ solution of $\left.(5.6): v_{T} \in D\left(A^{2}\right)\right\}$ satisfies

$$
\int_{0}^{1} v^{2}(0, x) \frac{1}{a} d x \leq \frac{1}{T_{1}-T_{0}} \int_{T_{0}}^{T_{1}} \int_{0}^{1} v^{2} \frac{1}{a} d x d t,
$$

for all $0<T_{0}<T_{1}<T$. Moreover, proceeding as in Lemma 4.3 and applying Proposition 5.2 , one has

$$
\int_{T_{0}}^{T_{1}} \int_{0}^{1} v^{2} \frac{1}{a} d x d t \leq C \int_{0}^{T} \int_{\omega} v^{2} \frac{1}{a} d x d t .
$$

for some positive constant $C$ and for all $v \in \mathcal{W}^{\prime}$.

Now, proceeding as in the proof of Proposition 4.1, one obtains the conclusion.

Finally, using Proposition 5.3 and a standard technique, one can extend the null controllability result established in Theorem 4.5:

THEOREM 5.4. Assume that the potential $b \in L^{\infty}(Q)$ and that Hypothesis 3.1 is satisfied. Then, given $T>0$ and $u_{0} \in L_{\frac{1}{a}}^{2}(0,1)$, there exists $f \in L^{2}(Q)$ such that the solution $u$ in $\mathcal{U}$ of (5.1) satisfies

$$
u(T, x)=0 \text { for every } x \in[0,1] .
$$

Moreover,

$$
\int_{Q} \chi_{\omega} f^{2} \frac{1}{a} d x d t \leq C \int_{0}^{1} u_{0}^{2} \frac{1}{a} d x
$$

for some positive constant $C$.

\subsection{A semilinear extension.}

In this subsection, as in [1], we will give, as a consequence of Theorem 5.4., the null controllability result for the following semilinear degenerate parabolic problem:

$$
\begin{cases}u_{t}-a(x) u_{x x}+h(t, x, u)=f(t, x) \chi_{\omega}(x), & (t, x) \in Q:=(0, T) \times(0,1), \\ u(t, 0)=u(t, 1)=0, & t \in(0, T), \\ u(0, x)=u_{0}(x), & x \in(0,1),\end{cases}
$$

where $a$ is as in Hypothesis 3.1 and the data $\left(f, u_{0}\right) \in L^{2}(Q) \times L_{\frac{1}{a}}^{2}(0,1)$. Moreover, we assume the following: 
HYPOTHESES 5.5. Let $h:[0, T] \times[0,1] \times \mathbb{R} \rightarrow \mathbb{R}$ be such that

$$
\begin{aligned}
& \forall \lambda \in \mathbb{R}, \quad(t, x) \mapsto h(t, x, \lambda) \text { is measurable, } \\
& \forall(t, x) \in(0, T) \times(0,1), \quad h(t, x, 0)=0,
\end{aligned}
$$

and there exist two positive constant $M$ and $C$ such that

$$
|h(t, x, \lambda)-h(t, x, \mu)| \leq M(1+|\lambda|+|\mu|)|\lambda-\mu|
$$

and

$$
\forall \lambda, \mu \in \mathbb{R}(h(t, x, \lambda+\mu)-h(t, x, \mu)) \lambda \geq-C \lambda^{2} .
$$

The previous assumptions on $h$ guarantee that for (5.8), Theorem 5.1. still holds (see [6]). However, for the well-posedness of (5.8) it is sufficient to require (5.12) with $\mu=0$, which is equivalent, thanks to (5.10)-(5.11), to the following apparently more general condition

$$
\begin{aligned}
\exists C \geq 0 \text { such that }-h(t, x, \lambda) \lambda \leq C\left(1+|\lambda|^{2}\right) \text { or, equivalently, } \\
-h(t, x, \lambda) \lambda \leq C|\lambda|^{2}
\end{aligned}
$$

(see [6]).

As in [1], using Theorem 5.4. applied to a suitable linear problem associated to (5.8), a standard fixed point method and the compactness of the embedding of $H_{0}^{1}(0,1)$ into $L^{2}(0,1)$ (recall that, by Corollary 2.7., $H_{0}^{1}(0,1)$ and $H_{\frac{1}{a}}^{1}(0,1)$ coincide), one can prove the null controllability property for (5.8) when $u_{0} \in H_{\frac{1}{a}}^{1}(0,1)$ and $f \in L^{2}(Q)$. Obviously, in this case the solutions of (5.8) and of the linear associated problem are more regular, i.e.,

$$
u \in H^{1}\left(0, T ; L_{\frac{1}{a}}^{2}(0,1)\right) \cap L^{2}(0, T ; D(A)) \cap C^{0}\left([0, T] ; H_{\frac{1}{a}}^{1}(0,1)\right) .
$$

Finally, as in [1] or as in [4], one can prove the null controllability result for (5.8) when $u_{0} \in L_{\frac{1}{a}}^{2}(0,1)$. In particular, one has:

THEOREM 5.6. Assume that Hypotheses 3.1 and 5.5. are satisfied. Then, given $T>0$ and $u_{0} \in L_{\frac{1}{a}}^{2}(0,1)$, there exists $f \in L^{2}(Q)$ such that the solution $u$ in $\mathcal{U}$ of (5.8) satisfies

$$
u(T, x)=0 \text { for every } x \in[0,1] .
$$

Moreover,

$$
\int_{Q} \chi_{\omega} f^{2} \frac{1}{a} d x d t \leq C \int_{0}^{1} u_{0}^{2} \frac{1}{a} d x
$$

for some positive constant $C$. 


\section{REFERENCES}

[1] Alabau-Boussouira, F., Cannarsa, P. and Fragnelli, G., Carleman estimates for degenerate parabolic operators with applications to null controllability J. Evol. Eqs 6 (2006), 161-204.

[2] Bensoussan, A., Da Prato, G., Delfout, M. C. and MitTer, S. K., Representation and Control of Infinite Dimensional Systems, Systems and Control: Foudations and applications, Birkhäuser, 1993.

[3] Barbu, V., FAVINI, A. and Romanelli S., Degenerate evolution equations and regularity of their associated semigroups, Funkcial. Ekvac 39 (1996), 421-448.

[4] CANNARsa, P. and Fragnelli, G., Null controllability of semilinear weakly degenerate parabolic equations in bounded domains, Electron. J. Diff. Eqns. 2006 (2006), no. 136, 1-20.

[5] Cannarsa, P., Fragnelli, G. and Vancostenoble, J., Linear degenerate parabolic equations in bounded domains: controllability and observability, Systems, control, modeling and optimization, edited by F. Ceragioli, A. Dontchev, H. Futura, K. Marti, and L.Pandolfi (Proceedings of the 22nd IFIP TC7 Conference held from July 18-33, 2005, in Turin, Italy), Springer, 163-173, 2006.

[6] Cannarsa, P., Fragnelli, G. and Vancostenoble, J., Regional controllability of semilinear degenerate parabolic equations in bounded domains, J. Math. Anal. Appl. 320 (2006), no. 2, 804-818.

[7] Cannarsa, P., Martinez, P. and Vancostenoble, J., Persistent regional controllability for a class of degenerate parabolic equations, Commun. Pure Appl. Anal. 3 (2004), 607-635.

[8] Cannarsa, P., Martinez, P. and Vancostenoble, J., Null controllability of the degenerate heat equations, Adv. Differential Equations 10 (2005), 153-190.

[9] Cannarsa, P., Martinez, P. and Vancostenoble, J., Carleman estimates for a class of degenerate parabolic operators, to appear in SIAM J. Control Optim.

[10] Davies E. B., Spectral Theory and Differential Operators, Cambridge Univ. Press. Cambridge, 1995

[11] ENGEL K. J. and NAGEL R., One-Parameter Semigroups for Linear Evolution Equations, Springer-Verlag, New York, Berlin, Heidelberg, 1999.

[12] Escauriaza, L., SERegin, G. and Sverak V., Backward uniqueness for parabolic equations, Arch. Ration. Mech. Anal. 169 (2003), no. 2, 147-157.

[13] ESCAURIAZA, L., SEREgIN, G. and SVERAK V., Backward uniqueness for the heat operator in half-space, St. Petersburg Math. J. 15 (2004), no. 1, 139-148.

[14] FATTORINI, H. O. AND RussELl, D. L., Exact controllability theorems for linear parabolic equations in one space dimension, Arch. Rat. Mech. Anal. 4 (1971), 272-292.

[15] FATTORINI, H. O., Infinite Dimensional Optimization and Control theory, Encyclopedia of Mathematics and its Applications, Cambridge University press, 1998.

[16] Favini, A. and Yagi, A., Degenerate Differential Equations in Banach Spaces, Pure and Applied Mathematics: A Series of Monographs and Textbooks 215, M.Dekker, New York, 1998.

[17] FELLER, W., The parabolic differential equations and the associated semigroups of transformations, Ann. of Math. 55 (1952), 468-519.

[18] Feller, W., Diffusion processes in one dimension, Trans. Am. Math. Soc. 97 (1954), 1-31.

[19] FERNÁNDEZ-CARA, E. and ZuAZUA, E., Null and approximate controllability for weakly blowing up semilinear heat equations, Ann. Inst. H. Poincaré Anal. Non Linéaire 17 (2000), no. 5, 583-616.

[20] Fursikov, A. V. and Imanuvilov, O. YU., Controllability of evolution equations, Lecture Notes Series 34, Research Institute of Mathematics, Global Analysis Research Center, Seoul National University, 1996.

[21] Karlin, S. and TAYlor, H. M., A Second Course in Stochastic Processes, Academic Press, 1981.

[22] Lebeau, G. and Robbiano, L., Contrôle exact de l'équation de la chaleur, Comm. P.D.E. 20 (1995), 335-356.

[23] Mandu, P., Analytical Treatment of One-Dimensional Markov Processes, Springer, New York, 1968.

[24] Martinez, P. and VANCOSTENOBLE, J., Carleman estimates for one dimensional degenerate heat equations, J. Evol. Eqs 6 (2006), 325-362.

[25] Martinez, P., RAYMOnd, J. P. and VAnCostenoble, J., Regional null controllability for a linearized Crocco type equation, SIAM J. Control Optim. 42 (2003), no. 2, 709-728.

[26] MICU, S. and ZUAZUA, E., On the lack of null controllability of the heat equation on the half-space, Portugaliae Math. 58 (2001), 1-24. 
[27] Micu, S. and ZuAZUA, E., On the lack of null controllability of the heat equation on the half-line, Trans. Amer. Math. Soc. 353 (2001), 1635-1659.

[28] RUSSELL, D. L., Controllability and stabilizability theorems for linear partial differential equations: recent progress and open questions, SIAM Review 20 (1978), 639-739.

[29] SHIMAKURA, N., Partial Differential Operators of elliptic type, Translations of Mathematical Monographs, 99, American Mathematical Society, Providence, RI, 1992.

[30] TAtaru, D., Carleman estimates, unique continuation and controllability for anizotropic PDE's, Contemporary Mathematics 209 (1997), 267-279.

\author{
Piermarco Cannarsa \\ Dipartimento di Matematica \\ Università degli Studi di Roma "Tor Vergata" \\ Via della Ricerca Scientifica, 00133 Roma \\ Italy \\ cannarsa@mat.uniroma2.it \\ Genni Fragnelli \\ Dipartimento di Ingegneria dell'Informazione \\ Università di Siena \\ Via Roma 56, 53100 Siena \\ Italy \\ fragnelli@dii.unisi.it \\ Dario Rocchetti \\ Dipartimento di Matematica \\ Università degli Studi di Roma "Tor Vergata" \\ Via della Ricerca Scientifica, 00133 Roma \\ Italy \\ rocchett@mat.uniroma2.it
}

